\title{
Prevalence of Diarrheal Disease Among Under-Five Children in Worabe Town, Southern Ethiopia
}

\author{
Aseb Arba*, Esayas Aydiko, Daniel Baza \\ School of Nursing, College of Medicine and Health Sciences, Wolaita Sodo University, Wolaita Sodo, Ethiopia \\ Email address: \\ 40aseb@gmail.com (A. Arba), esayasadako@gmail.com (E. Aydiko), danielbaza9@gmail.com (D. Baza) \\ ${ }^{*}$ Corresponding author
}

To cite this article:

Aseb Arba, Esayas Aydiko, Daniel Baza. Prevalence of Diarrheal Disease Among Under-Five Children in Worabe Town, Southern Ethiopia. American Journal of Life Sciences. Vol. 8, No. 4, 2020, pp. 45-51. doi: 10.11648/j.ajls.20200804.11

Received: July 23, 2020; Accepted: August 3, 2020; Published: August 13, 2020

\begin{abstract}
Background: Globally, childhood diarrheal diseases accounts for 18\% of childhood death. Diarrhea was most common among children of age 6-23 months. It is the second most common cause of child deaths next to pneumonia. Objective: To assess the determinants of diarrheal disease among under -five years of children in Worabe town Silte zone, Southern Ethiopia. Methodology: A community based cross-sectional study was conducted in 02 kebele, Worabe town from April to June 2018. Systematic random sampling method was used to get samples. Data was collected using questionnaire developed from different literatures. Data was analyzed by online bivariate analysis and p-value less than 0.05 was used to determine association. Result: A total of two hundred and twenty two respondents were included in the study. The prevalence of diarrhea among under-five children in 02 kebele was $69(30.9 \%)$. From the total 69 under-five children affected by diarrhea, $32(46.4 \%)$ were males and $37(53.6 \%)$ were females. There was significant association between diarrhea morbidity and age of the child $\left(\mathrm{X}^{2}=16.42(\mathrm{p}<0.05)\right)$, children feeding status (on exclusive breast feeding and complementary feeding $)\left(X^{2}=7.9009(p<0.05)\right)$, hand washing during preparing food for children $\left(X^{2}=49.55(p<0.001)\right)$, and solid waste disposal $\left(\mathrm{X}^{2}=21.56(\mathrm{p}<0.001)\right)$. Conclusion and Recommendation: The prevalence of diarrhea among under-five children in Worabe town was high. There was significant association between risk of having diarrhea with age of the child, exclusive breast feeding and complementary feeding, hand washing when preparing food for children, and solid waste disposal. Health Extension workers, woreda health office, kebele administrators and responsible bodies should educate communities on exclusive breast feeding, hygienic practices while preparing food and feeding children. Further research should be done using qualitative methods.
\end{abstract}

Keywords: Diarrhea, Diarrheal Diseases, Under-Five Children

\section{Introduction}

Diarrhea is passage of three or more loose or watery stool per 24 hours. It is one of the most important causes of morbidity and mortality in developing countries including in Ethiopia. In childhood, it accounts for $18 \%$ of deaths with an estimated deaths of 1.5 million children per year globally, making it the second most common cause of child death worldwide [1, 2]. World Health Organization (WHO) and United Nations International Children Emergency Fund (UNICEF) estimated that almost 2.5 billion episodes of diarrhea occur annually in children under five years of age in developing countries with more than $80 \%$ of the episodes occurring in Africa and South Asia [3, 4].
Diarrheal disease is the leading causes of death among under-five children. The overall diarrheal episode incidence is 3.2 per children per year. In developing countries, morbidity and mortality associated with childhood diarrhea remains challenging problem [4]. The dangers of diarrhea is related to complications like dehydration and malnutrition, while dysentery is another important cause of death due to fatal complication associated with it $[5,6]$.

Diarrheal disease is more common among rural children in SNNPR than children living in the other regions. Children living in Somali and Dire Dawa have the lowest prevalence of diarrhea when compared with children living in other region [7-9].

Most of diarrheal episodes occur in children in the first 
year of life. In some areas, young children spend $15-20 \%$ of their time with diarrheal illness. Diarrhea can last several days, and can leave the body without water and salt that are necessary for survival. Most children who die from diarrhea actually died from severe dehydration and fluid loss $[1,2$, $6]$.

It is widely recognized that exposure to diarrheal pathogens in developing countries is affected by a factors like age of the child, quality and quantity of water, availability of toilet facilities, housing conditions, maternal level of education, hose hold economic status, place of residence, feeding practices and general sanitary condition around the house [10-12].

Diarrheal illness can have a significant impact on psychomotor and cognitive development in young children. Early and repeated episode of childhood diarrhea complicated with malnutrition, co-infection, and anemia during a period of critical development, can have long term effects on linear growth as well as on physical and cognitive function [3].

Globally, mortality is declining but the overall diarrheal incidence remains unchanged and it is estimated to account for $13 \%$ of childhood disability adjusted life years [6]. In Ethiopia, the two weeks prevalence of diarrhea in underfive children is about $24 \%$. The Ethiopian Demographic and Health Survey 2016 report shows that diarrhea is a considerable child health problem; $12 \%$ of children under five were reported to have had diarrhea and $3 \%$ had diarrhea with blood in the two weeks before the survey and watery diarrhea was the commonest form. This study also shows that diarrhea was most common among children of age 6-23 months (23-25\%) with the prevalence varies seasonally. The prevalence of diarrheal disease among under five children residing both in Benishangul Gumuz and Gambella was both $23 \%$, but in SNNPR alone was $16.4 \%$ [7].

A study conducted in Tigray region on the patterns of childhood morbidity found that 3.05 diarrheal episodes occur per child per year [11]. Another study that attempted to determine household illness prevalence in Gondar showed that diarrhea was one of the most frequently occurring symptoms that account $11.4 \%$ of the overall complaint of seeking care [12]. The study conducted on under five children mortality due to acute watery diarrhea in GilgelGibe field was $30 \%$ [13].

According to the research conducted in Nekemte town, prevalence of diarrhea morbidity among under-five children was $28.9 \%$ [14]. Another survey in Mana district and Jimma town, revealed that the two week period of prevalence of childhood diarrhea morbidity was $33.7 \%$ and $36.5 \%$ respectively $[15,16]$. The analysis of reason of admission and death of under-five children to Swedish pediatric clinic in Addis Ababa, showed diarrheal disease accounts $21 \%$ of admission and $2.3 \%$ of deaths [17].

According to a follow-up study in Butajira, the incidence of diarrhea among under five children was about twoepisodes per person per year [18]. A community based study conducted in Keffa \& Sheka Zone found that the prevalence of diarrheal disease was $15 \%$ [15]. Diarrhea and malnutrition are known to have a bi-directional relationship that is they are potentially causing each other. Diarrhea may lead to malnutrition due to reduced dietary intake, mal absorption, and mal-digestion. On the other hand, malnutrition may cause and worsen diarrhea and other infections due to weekend immunity system [18-19].

There was limited information on the topic of interest in the study area. Therefore the objective of this study was to assess the prevalence of diarrheal disease and associated factors among under five children in Worabe town, Southern Ethiopia, 2018. The study will provide baseline information for those who are involved in prevention and control of diarrheal diseases.

\section{Methods and Materials}

\subsection{Study Setting and Period}

This study was conducted in Worabe town, Silte Zone, Southern Ethiopia. It is located some $172 \mathrm{~km}$ South West of Addis Ababa, the capital city of Ethiopia and $107 \mathrm{~km}$ from the Hawassa capital city of Southern Ethiopia. Regarding health services, in the town there are one governmental comprehensive and specialized hospital, one governmental health center and four private clinics.

\subsection{Study Design and Population}

A community based cross-sectional study design was used from, April-June, 2018. All households with under-five children living in Worabe town were taken as source population. All households with under-five children in 02 kebele of Worabe town were study population. Children under-five years of age whose mothers/caretakers were permanent residents of Worabe town included in study. Mothers who were unable to respond due to severely ill were excluded from study.

\subsection{Sample Size Calculation and Sampling Technique}

Sample size will be calculated by using single population formula, by taking proportion from EDHS 2011 report, the prevalence of diarrhea in SNNPR is about $16.4 \%$. Using correction formula, $\mathrm{n}=203$, adding $10 \%$ non-respondents, $\mathrm{n}$ $=223$, so final sample size is 223 .

Then, systematic sampling method was used after preparing sample frame to select study population. In households with more than one under five children, older one was included in sample.

\subsection{Variables}

Dependent variables: Diarrheal disease

Independent variables: Socio-demographic factors, feeding practice, economic status of family, educational status, source of water and storage and personal hygiene and environmental sanitation. 


\subsection{Data Collection Tool and Procedures}

Structured and pretested questionnaire and observational checklist were used to collect quantitative data. The questionnaire was developed after reviewing relevant literatures to the subject to include all the possible variables that address the objective of the study. The questionnaire was translated to local language and data was collected using it. After obtaining informed written consent, mother of the child was interviewed and condition of the environment was observed.

\subsection{Data Quality Control}

Brief training was given to data collectors and pretest was conducted before the actual study data collection. Based on pretest output, necessary corrections were made on tool. Onsite correction and strict supervision was made on data collectors.

\subsection{Data Analysis}

Descriptive analysis was made manually. Data was analyzed by online bivariate analysis and p-value less than 0.05 was used to determine association.

\subsection{Operational Definition}

Diarrhea -passage of three or more loose stool per 24 hours resulting excessive loss of fluid and electrolyte.

Acute diarrhea- diarrhea that begins acutely and terminate in less than two weeks.

Chronic diarrhea- diarrhea that stays beyond 2 weeks not necessarily occurs acutely.

Persistent diarrhea -diarrhea occurs acutely and lasts more than 2 weeks.

Cleanliness of the compound

Good: -ground of the compound is neat, use appropriate damping method, no feces and/or animal dung and other wastes seen in the compound, burn collected and decomposable wastes.

Fair: - if feces and other wastes are found in the compound, have pit to dispose waste, but do not burn it.

Poor: -if the compound is dirty, dispose waste on the open field, feces were there and full of wastes which is good provides best opportunity for breeding of insects and rodents breeding and does not meet either of criteria in good.

Cleanliness of the water container

Good: - if the water container is placed in clean area, inside of the container is clean, no fluid is leaking is seen and no other opening rather than normal opening, and has cover.

Fair:-inside of the container is clean, no fluid is leaking, and no opening is seen other than normal opening, but placed in unclean area.

Poor:-does not fulfill either of the above criteria.

\subsection{Ethical Consideration}

Approval to conduct this study was obtained from the Wolayta Sodo University, Department of Nursing. This study was conducted according to declaration of Helsinki, where study subjects were protected. Informed written consent was taken from respondents before interview. Respondents were informed that the responses kept as secrete and not be used for other purpose except for this study. Advice was given and referred the sick children to the near health institution. The name of either the respondent or the child was not recorded.

\section{Results}

\subsection{Socio-economic Characteristic Condition of the Households and Children}

A total of 223 households were interviewed with the response rate of $100 \%$. Concerning age of children of respondents, 35 (15.7\%) had children aged 0-6 months, 27 $(12.1 \%)$ had children in age group of 7-11 months, and 79 (35.4\%) had children aged between 36-59 months as shown in figure 1. Among the 223 respondents, 211 (94.6\%) were mothers and the rest $12(5.4 \%)$ were care takers to the child. From 223 respondents were 114 (51.1\%) male children and 109 (48.9\%) were females.

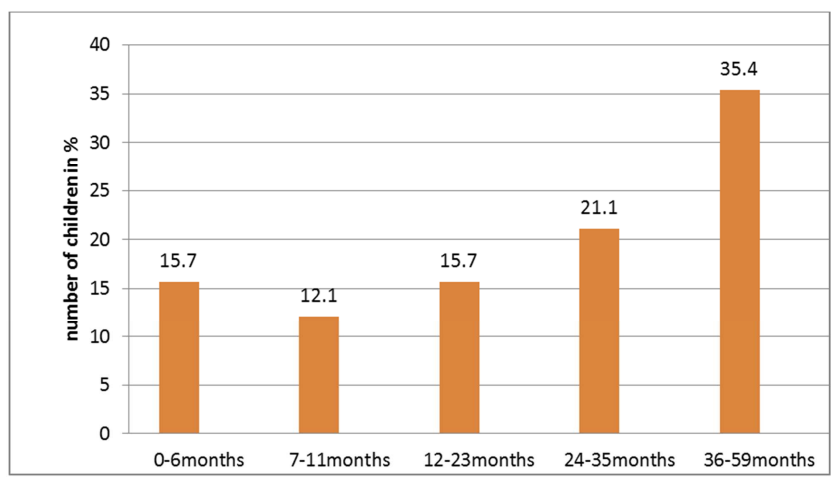

Figure 1. Number of under-five children in their age distribution in 02 kebele, Worabe town, Silte zone, SNNPR, June, 2018.

One hundred seventy seven $(79.4 \%)$ children born to mothers aged 18-34 years; whereas, $(20.6 \%)$ were born to mothers of age above 35 years. Concerning marital status of their mothers, 205 (91.9) were married, 9 (4\%) unmarried, 6 (2.8\%) divorced, and $3(1.3 \%)$ were widowed. From all, 191 (85.7\%) were Muslim, 19 (8.5\%) orthodox, and 13 (5.8\%) protestant. Majority of them were Silte $184(82.5 \%)$ in Ethnicity, followed by Amhara 13 (5.8\%). Concerning income of the family, $18(8.1 \%)$ of family were with average monthly income less than 500 Birr; 69 (30.9\%) had monthly income from 500 -1000birr and $136(61 \%)$ as shown in table 1.

Table 1. The socio-economic characteristics of the households with underfive children in 02 kebele in worabe town, Silte Zone, SNNPR, June 2018.

\begin{tabular}{llll}
\hline Variables & Responses & Frequency & Percentage (\%) \\
\hline \multirow{2}{*}{ Respondents } & Mother & 211 & 94.6 \\
& care taker & 12 & 5.4 \\
Marital status & Married & 205 & 91.9 \\
of the mother & Unmarried & 9 & 4 \\
\hline
\end{tabular}




\begin{tabular}{llll}
\hline Variables & Responses & Frequency & Percentage (\%) \\
\hline & Divorced & 6 & 2.7 \\
& Widowed & 3 & 1.3 \\
Religion of & Orthodox & 19 & 8.5 \\
mother & Protestant & 13 & 5.8 \\
& Muslim & 191 & 85.7 \\
Ethnic group of & Silte & 184 & 82.5 \\
mother & Gurage & 13 & 5.8 \\
& Other & 7 & 9.4 \\
Occupation of & House wife & 5 & 2.2 \\
mother & Government employ & 143 & 64.1 \\
& Merchant & 35 & 17.5 \\
Estimated & Other & 6 & 15.7 \\
household & $<500$ birr & 18 & 2.7 \\
income & 500-1000birr & 69 & 8.1 \\
\hline
\end{tabular}

\subsection{Educational Status of Mother}

Regarding education of mothers/ caretakers, 33 (14.8\%) of mothers cannot read and write (illiterate); whereas 52 $(23.3 \%)$ can read and write, 78 (35\%) learned up to elementary, and those who completed secondary, and above were $60(26.9 \%)$ as shown in figure 2 .

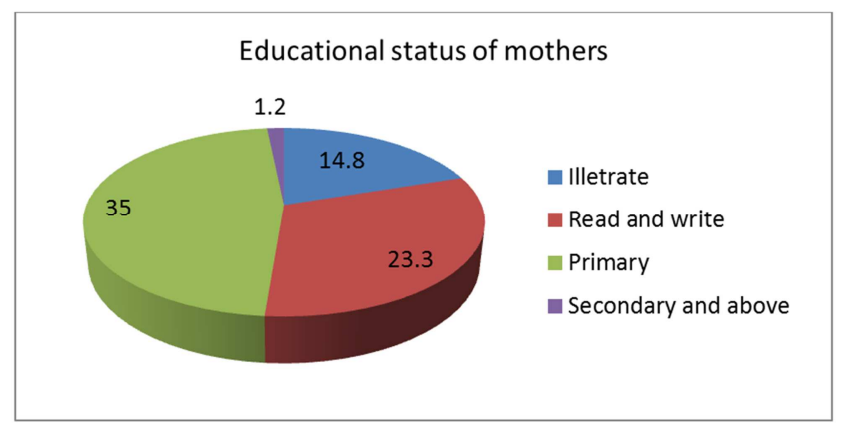

Figure 2. Educational level of mothers in 02 kebele, Worabe town, Silte zone, SNNPR, June, 2018 .

\subsection{Environmental Characteristics}

\section{Latrine condition}

From the total of 223 households observed, 220 (98.7\%) have latrine and the other $3(1.3 \%)$ didn't have latrine. From 220 households with latrine, $188(85.5 \%)$ have private latrines and the other $32(14.5 \%)$ share latrine with neighbors. Among 223 households, 8 (3.6\%) dispose wastes in waste disposal pit, $2(0.9 \%)$ dispose in open field, 155 $(69.5 \%)$ dispose in garbage receiver and the other $58(26 \%)$ dispose in municipality places as shown in table 2 .

Table 2. Latrine and waste disposal practice of 02 kebele households with under-five children in Worabe town, Silte zone, SNNPR, June, 2018.

\begin{tabular}{llll}
\hline & Responses & Frequency & Percentage \\
\hline \multirow{2}{*}{ Latrine } & Yes & 220 & 98.7 \\
& No & 3 & 1.3 \\
Ownership of latrine & Private & \urcorner 188 & 85.5 \\
& Shared & 32 & 14.5 \\
& Pit & 189 & 85.9 \\
Types of latrine & Flash & 9 & 4.1 \\
& Ventilated & 22 & 10 \\
\hline
\end{tabular}

\begin{tabular}{llll}
\hline & Responses & Frequency & Percentage \\
\hline \multirow{4}{*}{ Waste disposal } & Pit & 8 & 3.6 \\
& Open field & 2 & 0.9 \\
& Garbage can & 155 & 69.5 \\
& Municipality & 58 & 26 \\
\hline
\end{tabular}

\subsection{Water Utilization}

Among 223 households interviewed, 206 (92.4\%) got their drinking water from pipe, $11(4.9 \%)$ got from protected sources/wells, 6 (2.7\%) from unprotected sources. From those, 177 (79.4\%) store the drinking water using Jerican, 29 (13\%) store in bucket with lid and 17 household (7.6\%) store using other equipment. All mothers transport the drinking water from the source to home using covered container as shown in table 3 below.

Table 3. The water utilization practice among households with under-five children in 02 kebele, worabe Town, Silte Zone, SNNPR, June 2018.

\begin{tabular}{llll}
\hline Variables & Categories & Frequency & Percentage \\
\hline & Pipe & 206 & 92.4 \\
Drinking water & Protected source & 11 & 4.9 \\
& Unprotected source & 6 & 2.7 \\
& River & 0 & 0 \\
& Jeri can & 177 & 79.4 \\
Storage of drinking & Bucket with lid & 29 & 13 \\
water at home & Open bucket & 0 & 0 \\
& Other & 17 & 7.6 \\
The time spent to & $<15$ minutes & 208 & 92.3 \\
fetch water & $\geq 15$ minutes & 15 & 6.7 \\
Amount of water & $<20$ Liters & 64 & 28.7 \\
utilized for & $\geq 20$ Liters & 159 & 71.3 \\
household daily? & & & \\
\hline
\end{tabular}

\subsection{Child Feeding Practice}

From 223 children, $36(16.1 \%)$ were on exclusive breast feeding but the other $187(83.9 \%)$ were taking additional food other than breast. For those children on complementary feeding, $25(13.4 \%)$ mothers use their hand to feed their child, 82 (48.9\%) use bottle and, 57 (30.5\%) use cup, and 22 (11.8\%) mothers use other (spoon).

Among 223 children, 114 (51.1\%) were males and the other 109 (48.9) were females. Among them, 69 (30.9\%) had diarrhea in the past four weeks before data collection. From those who had diarrhea, 41 (51.25\%) children had experienced 3 episodes, 34 (42.5\%) had experienced more than 3 episodes and for the other 5 children (6.5\%) mothers didn't know how many episodes were they experienced.

Among children experienced diarrhea 61 (88.4\%) affected by diarrhea less than 14 days and rest $8(11.6 \%)$ affected by diarrhea $\geq 14$ days before surveys. Of the affected children $52(75.4 \%)$ had acute watery diarrhea and $10(14.5 \%)$ had had blood diarrhea and $7(10.1 \%)$ had mucoid diarrhea. 66 $(95.7 \%)$ children were taken to the health institution, and 3 $(4.3 \%)$ children were given home based treatment as displayed in table 4 . 


\subsection{Bivariate Analysis Result}

There was association between age of children $X^{2}=16.42$ $(p=0.00251)$, exclusive breast feeding and complementary feeding $\mathrm{X}^{2}=7.9009(\mathrm{P}, 0.0494)$, hand washing when preparing food for child $\mathrm{X}^{2}=49.55(\mathrm{P}=0.00001)$, waste disposal practice $\mathrm{X}^{2}=21.56(\mathrm{P}, 0.000081)$ and risk of having under-five diarrheal disease. There was no significant association between sex of children $X^{2}=0.9(p=0.3428)$, educational level of mother $\mathrm{X}^{2}=7.7704(\mathrm{P}, 0.051)$, monthly family income $X^{2}=1.93(0.3809)$ and risk of having diarrheal disease among under five children in study area as shown in table 4.

Table 4. Bivariate analysis result on predictors of diarrheal disease among under-five children in 02 Kebele, Worabe town, Silte zone, June, 2018.

\begin{tabular}{|c|c|c|c|c|}
\hline \multirow{2}{*}{ Variables } & \multirow{2}{*}{ Category } & \multicolumn{2}{|c|}{ Diarrhea } & \multirow{2}{*}{$X^{2}$ (p-value) } \\
\hline & & Yes & No & \\
\hline \multirow{5}{*}{ Age of children in (months) } & $0-6$ & 3 & 32 & \multirow{5}{*}{$16.42(0.00251)^{*}$} \\
\hline & $7-11$ & 15 & 12 & \\
\hline & $12-23$ & 12 & 23 & \\
\hline & 24-35 & 13 & 34 & \\
\hline & $36-59$ & 26 & 53 & \\
\hline \multirow{2}{*}{ Sex of child } & Male & 32 & 82 & \multirow{2}{*}{$0.9(0.3428)$} \\
\hline & Female & 37 & 72 & \\
\hline \multirow{3}{*}{ Wash of hands when preparing food. } & Before preparing food & 7 & 32 & \multirow{3}{*}{$49.55(0.00001)^{*}$} \\
\hline & After preparing food & 22 & 1 & \\
\hline & Before \& after preparing food & 40 & 121 & \\
\hline \multirow{2}{*}{ Childs current status } & Only breast feed & 4 & 32 & \multirow{2}{*}{$7.9009(0.0494)^{*}$} \\
\hline & Complementary feeding & 65 & 122 & \\
\hline \multirow{4}{*}{ Educational level of mother } & Illiterate & 16 & 17 & \multirow{4}{*}{$7.7704(0.051)$} \\
\hline & Read and write & 14 & 38 & \\
\hline & Primary & 26 & 52 & \\
\hline & Secondary and above twelve & 13 & 47 & \\
\hline \multirow{3}{*}{ Household income } & $<500$ birr & 3 & 15 & \multirow{3}{*}{$1.93(0.3809)$} \\
\hline & 500-1000birr & 23 & 46 & \\
\hline & >1000birr & 43 & 93 & \\
\hline \multirow{2}{*}{ Latrine } & Yes & 67 & 153 & \multirow{2}{*}{$1.8163(0.17776)$} \\
\hline & No & 2 & 1 & \\
\hline \multirow{4}{*}{ Solid waste disposal } & Pit & 7 & 1 & \multirow{4}{*}{$21.56(0.000081)^{*}$} \\
\hline & Open field & 1 & 1 & \\
\hline & garbage bin & 35 & 120 & \\
\hline & Municipality & 25 & 33 & \\
\hline \multirow{3}{*}{ Drinking water } & Pipe & 58 & 148 & \multirow{3}{*}{$2.842(\mathrm{P}, 0.2414)$} \\
\hline & Protected source & 5 & 6 & \\
\hline & Unprotected source & 6 & 0 & \\
\hline \multirow{3}{*}{ Store of drinking water } & Jeri can & 53 & 124 & \multirow{3}{*}{$2.302[0.3163]$} \\
\hline & Bucket with lid & 8 & 21 & \\
\hline & Other & 8 & 9 & \\
\hline \multirow{2}{*}{ Amount of water utilized daily } & $<20$ lit & 17 & 47 & \\
\hline & $>20$ lit & 52 & 107 & $0.8056[0.3694]$ \\
\hline
\end{tabular}

* -presence of association

\section{Discussion}

This study revealed that the prevalence of diarrheal disease among under-five children 4 was $30.9 \%$. This figure is high compared the prevalence of diarrhea in SNNPR (16.4\%), Nekemt town $(28.9 \%)$, Kefa Sheka zone $(15 \%)$ but it is relatively comparable with the finding from Mana district (33.7\%) and lower than the finding in Jima town and (36.5\%), Senegal and Liberia (each 40\%) [8, 11-16]. The analysis of 73 comprehensive studies in sub Saharan African countries showed that prevalence of diarrhea ranges from $10.5 \%-19 \%$ [19]. This could be due to the difference in the community composition in different parameters.
This study showed that there was significant association between age of children and risk of having diarrhea $16.42(\mathrm{P}$, $0.00251)$. The most commonly affected age group was $35-59$ months. This is consistent with the research finding to longitudinal community based in sub-Saharan Africa [19].

There was significant association between exclusive breast feeding and complementary feeding and risk of having diarrhea under-five children in this study. This is consistent with the analysis on breast feeding and risk of diarrhea indicated that the risk of developing diarrheal disease in partially breast feed infants was five times higher than that of infants exclusively on breast milk [19].

There was significant association between hand washing 
when preparing food for child and solid waste disposal and risk of having diarrhea in this study. This study was supported by study conducted in Nekemte town. This could be due to the fact that food contamination source includes unclean hands, feces, polluted water, flies, pests, domestic animals, unclean utensils, and pots, and unsanitary environment [14].

As it was revealed this study, there was no significant association between educational level of mother and risk of having diarrhea for under- five children. This is consistent with the study conducted in the republic of Congo which showed that highly educated mothers reported less- diarrhea [14].

This study showed that there was no significant association between drinking water and amount of water utilization per day and risk of having diarrhea in under- five children. This is consistent with the study conducted in Republic Congo showed that children coming from households that obtain water from protected source were less likely to have diarrhea as compared to those who get water supply from unprotected source [15].

There was no association between presences of latrine and risk of diarrhea in this study. This is different from the study conducted in republic Congo which shows that children of family with latrine had lower prevalence of diarrhea than those children whose families didn't have latrine [15]. This is may be due to the number of households that are included in this study with no latrine are small in number or social bias or desirability of respondents' to give information.

\section{Limitations}

We used cross-sectional study design, which couldn't determine cause-effect relationship. There may be social desirability and recall bias that affect information obtained.

\section{Conclusions}

In general, prevalence of diarrhea among under-five children in Worabe town was high. The highest occurrence of diarrheal disease was seen among children aged 36-59 months. Age of child, appropriate exclusive breast feeding practice and complementary feeding practice, hand washing when preparing food for child and proper solid waste disposal system were factors associated with under-five diarrhea.

\section{Recommendations}

Based on the finding of study, Worabe Town administrator, Non-governmental Organizations, Health extension workers and Health care providers should give the awareness and empower mothers on how to care their children with minimizing on avoiding transmission of microorganisms. Further study is recommended with inclusion of qualitative methods of data collection and analysis..

\section{Declaration}

1) Ethics approval: This study was approved by Wolaita Sodo University College of Medicine and Health Sciences Ethics Review Committee. Informed voluntary written consent was obtained prior to interview.

2) Consent to participate and consent for publication: Mothers of under five children gave written informed consent to participate and consent for publication.

3) Availability of data and material: The datasets of current study are available with the requests to corresponding author to access data concerning this study.

4) Competing interests: The authors declare they have no conflict of interest

5) Funding: Funding for this study was self-sponsored by Authors

6) Authors' contributions:

a) Aseb Arba: Idea generation, methodology, writing, funding, analysis and final write up

b) Esayas Aydiko: Introduction, methodology and funding

c) Daniel Baza: Introduction, methodology and funding

7) Acknowledgements: Heartfelt thanks message deserves for mothers for their participation, kebele administrators

\section{References}

[1] Robert M, Bonita F, Joseph W, Nina F and Richard E. Nelson text book of pediatrics, $19^{\text {th }}$ ed. New York: Elsevier; 2011.

[2] Pervez Akeber Khan. Infectious disease. Basis of pediatrics $6^{\text {th }}$ edition. Nishtor medical college, India 346-451.

[3] WHO. Childhood disease in Africa. fact sheet, Http://www.Who.inf/inf.fs/fact log.html

[4] African demographic and health survey 1992. The prevalence of diarrheal in under five children in Boteswana.

[5] Timaeus IM \& Lush L. Intra urban differential in child death. 1995; 5: 163-190.

[6] www.mediainquiries@WHO.int.WHO media center.

[7] Central Statistical Authority, Ethiopian demographic and health survey, 2016. Addis Ababa and Calverton, Maryland, USA.

[8] Belachew T, Jira C, Faris K, Mekete G, Asres T, and Aragaw $\mathrm{H}$, Diarrheal Disease for the Ethiopian Health Center Team, Jimma University In collaboration with the Ethiopia Public Health Training Initiative and the Carter Center, in 2004.

[9] Central Statistical Authority, Ethiopian demographic and health survey, 2011. Addis Ababa and Calverton, Maryland, USA.

[10] DN. Gubta. Nature and significance of diarrhea in the rural community among children below 5 years. National institute of cholera and enteric diseases, India.

[11] Ali M., Asfaw T, Beyene H., Bypass P., Hisabu MS\& Pederson FK. A community based study of childhood morbidity in Tigray, Northern Ethiopia, J Health Dev, 2001; 15 (3): 165-172.21.

[12] Mitike G. Prevalence of acute and persistent diarrhea in North Gondar zone, Ethiopia. East Africa, med. J. aug, 2001; 78 (8): 44-48. 
[13] Amare D, Fasil T, Belayneh G. Determinants of under-five mortality in Gilgel Gibe field research center, south west Ethiopia. Ethiopian journal of health development; 2007; 21 (2): 117.

[14] Wondwossen B. A stepwise regression analysis on under-five morbidity prevalence in Nekemte town, western Ethiopia. Maternal care giving and hygiene behavioral determinants. East African Journal of public health; 2008; 5 (3): 193-98.

[15] Teklemariam S, Getaneh $\mathrm{T}$ \& Bekele F. Environmental determinants of diarrhea morbidity in under five children, keffa- sheka zone, South west Ethiopia. Ethiop, med, J, 2000; 38 (1): 27-34.
[16] Getaneh T, Assefa A, Tadesse Z.; Diarrhea morbidity in urban areas of south west Ethiopia. East African Med. Journal; 1997: 74 (8): 491-494.

[17] Mock NB, Sellers TA, Abdoh AA \& Franklin RR. Socioeconomic, environmental, demographic and behavioral factors associated with the occurrence of diarrhea in young children. Soc. Sci. Med. 1993; 36 (6): 807-8.

[18] Ketsela T. Knowledge and practice of mothers or care takers towards diarrhea and its treatment in rural communities in Ethiopia. Ethiopian medical journal, 1991; 29 (4).

[19] Child health research project, childhood disease in SubSaharan Africa special report, April 1998; 2 (1). 